\title{
Correction: The newborn metabolome: associations with gestational diabetes, sex, gestation, birth mode, and birth weight
}

Toby Mansell, Amanda Vlahos, Fiona Collier, Anne-Louise Ponsonby, Peter Vuillermin, Susan Ellul, Mimi L. K. Tang, David Burgner, Richard Saffery on behalf of the Barwon Infant Study Investigator team*

(c) The Author(s), under exclusive licence to the International Pediatric Research Foundation, Inc 2021

Pediatric Research (2022) 91:1915-1916; https://doi.org/10.1038/s41390-021-01789-9

Correction to: Pediatric Research https://doi.org/10.1038/s41390021-01672-7, published online 15 September 2021

In this article, the legend for Fig. 2 was inadvertently misrepresented; the figure should appear as shown below. The original article has been corrected.
Publisher's Note Springer Nature remains neutral with regard to jurisdictional claims in published maps and institutional affiliations. 


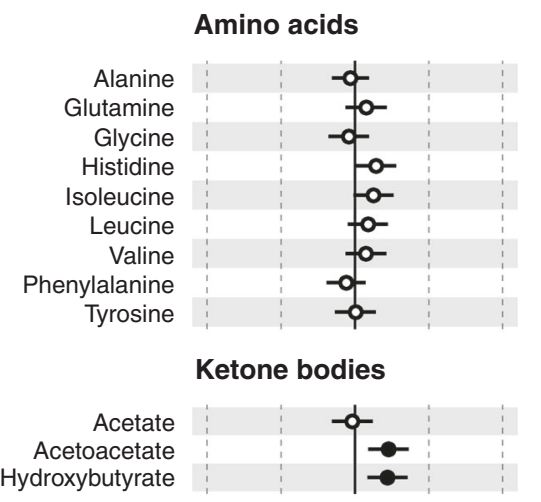

Glycerides and phospholipids

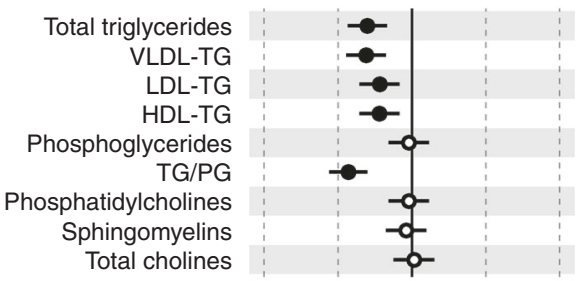

Glycolysis related metabolites

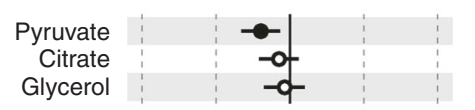

Fluid balance



b

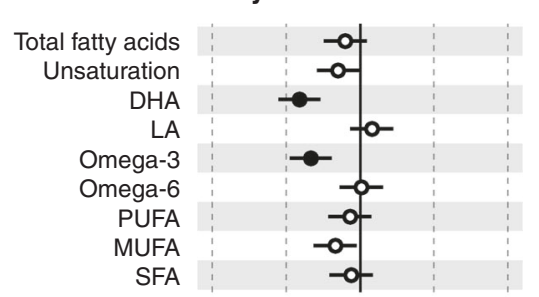

Apolipoproteins

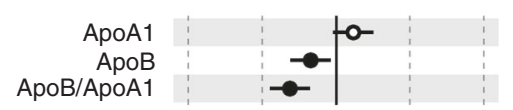

Cholesterol

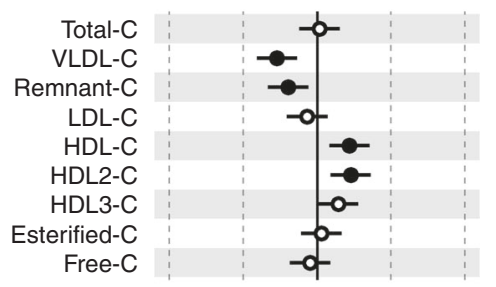

Lipoprotein particle sizes

VLDL particle size LDL particle size HDL particle size

\section{Inflammation}

Glycoprotein acetyls

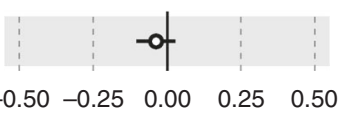

Difference in birth log biomarkers (SD units) per 1-SD increase in birth weight $z$-score (adj. for sex,

gest. age, and mode of delivery)

Fig. 2 Birth weight z-score and cord blood serum NMR metabolomic profiles. The difference in mean metabolite log concentration in SD units per 1 unit increase in birth weight $z$-score in adjusted models. Panel (a) is amino acids, ketone bodies, glycerides and phospholipids, glycolysis-related metabolites, and fluid balance markers; (b) is fatty acids, apolipoproteins, cholesterol, lipoprotein particle size, and inflammation (GlycA). Error bars represent $95 \%$ confidence intervals. Closed points represent associations with significant $p$ value at the $5 \%$ FDR level after adjustment using the Benjamini-Hochberg method. The associations depicted here are listed in Supplementary Table S5. C cholesterol, DHA docosahexaenoic acid, HDL high-density lipoprotein, IDL intermediate density lipoprotein, LA linoleic acid, LDL low-density lipoprotein, MUFA monounsaturated fatty acids, PG phosphoglycerides, PUFA polyunsaturated fatty acids, SFA saturated fatty acids, TG triglycerides, VLDL very-low-density lipoprotein. 